\title{
Potential Biomarkers for Ovarian Cancer
}

\author{
Maria Rosa Maduro, PhD
}

Reproductive Sciences

2019, Vol. 26(4) 449

(C) The Author(s) 2019

Article reuse guidelines:

sagepub.com/journals-permissions

DOI: $10.1177 / 1933719119833449$

journals.sagepub.com/home/rsx

SAGE
Ovarian cancer constitutes the fifth most common cause of cancer deaths among women. It is estimated that 239000 new cases are diagnosed all over the world every year, with 152000 deaths being reported worldwide annually as a result of ovarian cancer. ${ }^{1}$ Part of the mortality associated with ovarian cancer comes from the difficulty in diagnosing the disease at its early stages when treatment has a greater chance of success, mostly due to the nature of early symptoms that are often unspecific or even absent. ${ }^{2}$ Therefore, the need for diagnostic tools that can detect early and efficiently this devastating disease is a medical, social, and economic priority. Thus, many studies have focused on better understanding the disease in order to identify not only new ways of treatment but also new potential markers of early ovarian cancer stages. ${ }^{3-8}$

In the present issue of Reproductive Sciences, Záveský and colleagues report the results of their study that aimed to explore and evaluate the expression of extracellular microRNAs (miRNAs) in the excessive fluid buildup often manifested in ovarian cancer (known as ascites), to determine possible associations with disease characteristics, cancer progression, and patient outcome. ${ }^{9}$

In this study, Záveský et al performed large-scale expression profiling of 754 human miRNAs, using reverse transcriptase quantitative polymerase chain reaction and 384-well TaqMan Array Human MicroRNA A and B cards, in order to identify differentially expressed miRNAs between the extracellular fraction of the ascitic fluid and the associated high-grade serous ovarian carcinomas and control plasma.

The authors observed that 153 miRNAs out of the ones analyzed were differently expressed relative to the controls in a significant matter. Of these, 7 individual miRNAs (miR-200a, b, and c, miR-141, miR-429, miR-1290, and miR-30a-5p) were further tested in extended sample sets including serous, endometrial, and mucinous subtypes of cancer.

Záveský and colleagues noted that all miR-200 family members, along with miR-1290, were notably overexpressed, while miR-30a5p showed only a weak overexpression. In addition, their receiver operating characteristic curve analyses indicated that miRNA expression was able to successful discriminate pathological samples from controls. Furthermore, the authors observed that high expression levels of miR-200b were associated with longer overall survival, while low expression levels were correlated with poor overall survival.

In sum, the work presented by Záveský and colleagues indicates that extracellular miRNAs derived from ascites may be good candidates for prognostic biomarkers of ovarian cancer. Additionally, their findings reinforce the general idea that miRNAs are posttranscriptional regulators of gene expression with promising potential in cancer diagnostics and treatment.

\section{References}

1. Reid BM, Permuth JB, Sellers TA. Epidemiology of ovarian cancer: a review. Cancer Biol Med. 2017;14(1):9-32.

2. Wei W, Li N, Sun Y, Li B, Xu L, Wu L. Clinical outcome and prognostic factors of patients with early stage epithelial ovarian cancer. Oncotarget. 2017;8(14):23862-23870.

3. Wang Y, Zhang W, Wang X, et al. Expression of Sam68 correlates with cell proliferation and survival in epithelial ovarian cancer. Reprod Sci. 2017;24(1):97-108.

4. Samtani R, Sharma N, Garg D. Effects of endocrine-disrupting chemicals and epigenetic modifications in ovarian cancer: a review. Reprod Sci. 2018;25(1):7-18.

5. Gu A, Jie Y, Yao Q, Zhang Y, Mingyan E. Slug is associated with tumor metastasis and angiogenesis in ovarian cancer. Reprod Sci. 2017;24(2):291-299.

6. Kwon Y, Godwin AK. Regulation of HGF and c-MET interaction in normal ovary and ovarian cancer. Reprod Sci. 2017;24(4): 494-501.

7. An Q, Zhou Y, Han C, Zhou Y, Li F, Li D. BTG3 overexpression suppresses the proliferation and invasion in epithelial ovarian cancer cell by regulating AKT/GSK3 $\beta / \beta$-catenin signaling. Reprod Sci. 2017;24(10):1462-1468.

8. Yildirim N, Kocal GC, Isik Z, et al. Ubiquitin-proteasome axis, especially ubiquitin-specific protease-17 (USP17) gene family, is a potential target for epithelial-mesenchymal transition in high-grade serous ovarian cancer [Published online]. Reprod Sci. 2018: 1933719118799189. doi:10.1177/1933719118799189

9. Záveský L, Jandáková E, Weinberger V, et al. Ascites-derived extracellular microRNAs as potential biomarkers for ovarian cancer. Reprod Sci. 2019;26(4):510-522. 